\title{
Properties of individual and integrated pulses drawn into mode changing phenomenon in PSR 0943+10 at 40, 103 and $430 \mathrm{MHz}$
}

\author{
S.A. Suleymanova, V.A. Izvekova \\ Astrospace Center of Lebedev Physics Institute, Russia \\ J.M.Rankin \\ University of Vermont, USA
}

\begin{abstract}
Pulsar $0943+10$ demonstrates very obviously that the mode switching phenomenon includes simultaneous and dramatic alterations of all principal properties of radioemission, i.e. intensity, polarization (both linear and circular) and drifting behaviour of individual pulses over a broad frequency range. On the basis of observations of PSR 0943+10 at the frequencies 40 and $430 \mathrm{MHz}$, a complete scenario of the transition process between two modes of radiation has been revealed. The "pre-switching transition process" is found which manifests itself as attenuation of the pulse intensity of the current mode during hundreds of the pulse periods preceding a switch.
\end{abstract}

\section{Results and discussion}

Pulsar PSR 0943+10 is known as a mode-switcher at $103 \mathrm{MHz}$ (Suleymanova and Izvekova, 1984).

A long train of 986 pulses at $430 \mathrm{MHz}$ detected in October 1992 at Arecibo (USA) were analyzed together with several shorter sets of pulses at 40 and 103 $\mathrm{MHz}$ observed at Pushchino, (Russia) in 90-s. This allowed to reveal two distinct shapes of integrated profiles at 40 and $430 \mathrm{MHz}$. At $40 \mathrm{MHz}$ integrated intensity profile in B mode has two resolved components A and B (Figure 1, left upper panel) while $\mathrm{Q}$ mode profile (dashed line) has components $\mathrm{A}$ and $\mathrm{C}$ (central). The component $\mathrm{C}$ is shifted by 4.5 degrees to the earlier longitudes from component B. At $430 \mathrm{MHz}$ pulse window, polarization state and drifting behaviour of individual pulses have been changed dramatically after pulse number 817 . It has been interpreted as mode switching event. Intensity profile in B mode at $430 \mathrm{MHz}$ (pulses 1-817 were summarized) has only one component $A$ (Figure 1, left lower panel). In Q mode intensity profile components A, B and $\mathrm{C}$ are not resolved (dashed line).

It is found that linear polarization angle distribution changes significantly and in similar way at 40,103 , and $430 \mathrm{MHz}$ together with integrated pulse shape alterations (Suleymanova et al., 1996). The integrated circular polarization profile is affected by mode switching as well. 

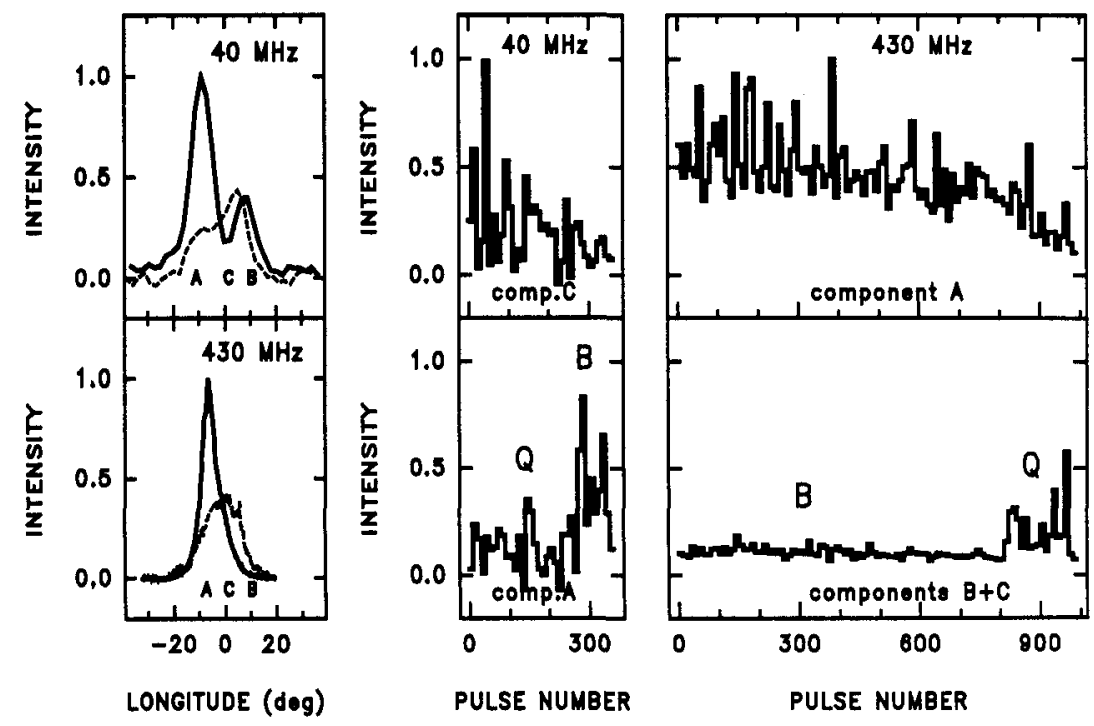

Figure 1. Intensity profiles (left panel) and time variation in the intensity of individual pulses from PSR $0943+10$ in three windows: A, $\mathrm{B}$ and $\mathrm{C}$ at 40 (central panel) and $430 \mathrm{MHz}$ (right panel).

We studied a switch from $\mathrm{Q}$ mode to $\mathrm{B}$ mode at $40 \mathrm{MHz}$ and from $\mathrm{B}$ to $\mathrm{Q}$ mode at $430 \mathrm{MHz}$. Figure 1 (central and right panels) shows time variation in the intensity of individual pulses from PSR $0943+10$ in three longitude ranges: $\mathrm{A}, \mathrm{B}$ and $\mathrm{C}$ at 40 and $430 \mathrm{MHz}$.

The "pre-switching transition process" is found, which manifests itself as attenuation of the pulse intensity of the current mode during hundreds of the pulse periods. This attenuation goes into fast reduction of intensity beginning a minute before and during some time after the moment when an alternative mode switches on.

Thus, the mode switching phenomenon in PSR 0943+10 contains two time scales: first scale less than one pulsar period shows that alternative mode switches on instantaneously and the second one which is of about several hundreds of periods shows that switching on is preceeded by slow falling down of the current mode activity.

Acknowledgments. This work was supported by Russian Federal Scientific Program "Astronomy" (Project 3-180) and INTAS (Grant INTAS-94-3097).

\section{References}

Suleymanova, S.A., Izvekova, V.A., Pugachev, V.D. 1996, Proceedings IAU 160 Suleymanova, S.A., Izvekova, V.A. 1984, Soviet Ast., 28, 32 